RECEIVED

DOE/SF/19460--299-Pt.8 MAY O 6999

OSTI
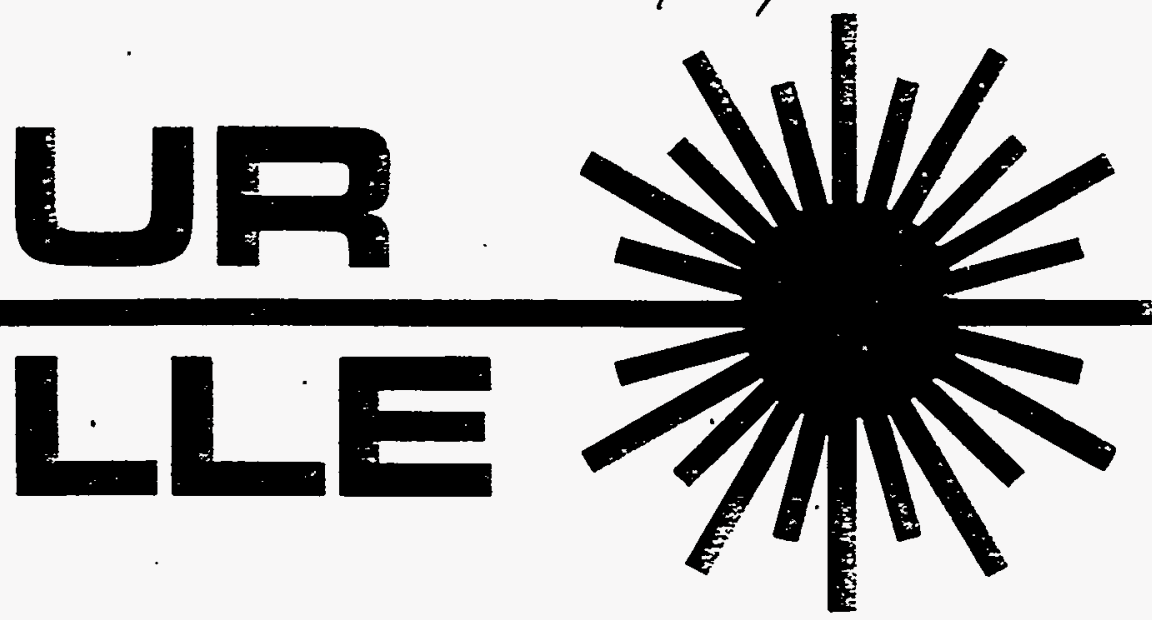

1998 SUMMER RESEARCH PROGRAM FOR HIGH SCHOOL JUNIORS

AT THE

UNIVERSITY OF ROCHESTER'S

LABORATORY FOR LASER ENERGETICS

STUDENT RESEARCH REPORTS

PROJECT COORDINATOR

Dr. R. Stephen Craxton

March 1999

DISTAIBUTION OF THIS DOCUMENT IS UANATEO

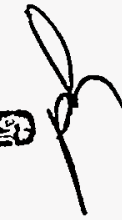

Laboratory Report 300

MASTER

Laboratory for Laser Energetics University of Rocnester 


\section{DISCLAIMER}

This report was prepared as an account of work sponsored by an agency of the United States Government. Neither the United States Government nor any agency thereof, nor any of their employees, makes any warranty, express or implied, or assumes any legal liability or responsibility for the accuracy, completeness, or usefulness of any information, apparatus, product, or process disclosed, or represents that its use would not infringe privately owned rights. Reference herein to any specific commercial product, process, or service by trade name, trademark, manufacturer, or otherwise does not necessarily constitute or imply its endorsement, recommendation, or favoring by the United States Government or any agency thereof. The views and opinions of authors expressed herein do not necessarily state or reflect those of the United States Government or any agency thereof. 


\section{DISCLAIMER}

Portions of this document may be illegible in electronic image products. Images are produced from the best available original document. 


\title{
1998 SUMMER RESEARCH PROGRAM FOR HIGH SCHOOL JUNIORS \\ AT THE \\ UNIVERSITY OF ROCHESTER'S \\ LABORATORY FOR LASER ENERGETICS
}

\author{
STUDENT RESEARCH REPORTS
}

\section{PROGRAM COORDINATOR}

\author{
Dr. R. Stephen Craxton \\ LABORATORY FOR LASER ENERGETICS \\ University of Rochester \\ 250 East River Road \\ Rochester, NY 14623-1299
}

During the summer of 1998. 11 students from Rochester-area high schools participated in the Laboratory for Laser Energetics' Summer High School Research Program. The goal of this program is to excite a group of high school students about careers in the areas of science and technology by exposing them to research in a state-ofthe-art environment. Too often, students are exposed to "research" only through classroom laboratories that have prescribed procedures and predictable results. In LLE's summer program, the students experience all of the trials, tribulations, and rewards of scientific research. By participating in research in a real environment, the students often 
become more excited about careers in science and technology. In addition, LLE gains from the contributions of the many highly talented students who are attracted to the program.

The students spent most of their time working on their individual research projects with members of LLE's technical staff. The projects were related to current research activities at LLE and covered a broad range of areas of interest including optics, spectroscopy, chemistry, diagnostic development, and materials science. The students, their high schools, their LLE supervisors and their project titles are listed in the table. Their written reports are collected in this volume.

The students attended weekly seminars on technical topics associated with LLE's research. Topics this year included lasers, fusion, holography, nonlinear optics, global warming, and scientific ethics. The students also received safety training, learned how to give scientific presentations, and were introduced to LLE's resources, especially the computational facilities.

The program culminated with the High School Student Summer Research Symposium on 26 August at which the students presented the results of their research to an audience that included parents, teachers, and members of LLE. Each student spoke for approximately ten minutes and answered questions. At the symposium an Inspirational Science Teacher award was presented to Mr. David Crane, a chemistry teacher at Greece Arcadia High School. This annual award honors a teacher, nominated by alumni of the LLE program, who has inspired outstanding students in the areas of science, mathematics, and technology. 


\begin{tabular}{|c|c|c|c|}
\hline \multicolumn{4}{|c|}{ High School Students and Their Projects (1998) } \\
\hline Student & High School & Supervisor & Project \\
\hline Steven Corsello & Pittsford Mendon & K. Marshall & $\begin{array}{l}\text { Computer-Aided Design and } \\
\text { Modeling of Nickel Dithiolene } \\
\text { Near-Infrared Dyes }\end{array}$ \\
\hline Peter Grossman & Wilson Magnet & R. S. Craxton & $\begin{array}{l}\text { Group Velocity Effects in } \\
\text { Broadband Frequency } \\
\text { Conversion on OMEGA }\end{array}$ \\
\hline Joshua Hubregsen & Pittsford Sutherland & S. Jacobs & $\begin{array}{l}\text { A Study of Material Removal } \\
\text { During Magnetorheological } \\
\text { Finishing (MRF) }\end{array}$ \\
\hline Nieraj Jain & Pittsford Sutherland & M. Guardelben & $\begin{array}{l}\text { Analyzing Algorithms for } \\
\text { Nonlinear and Spatially } \\
\text { Nonuniform Phase Shifts in } \\
\text { the Liquid Crystal Point } \\
\text { Diffraction Interferometer }\end{array}$ \\
\hline Leslie Lai & Pittsford Mendon & M. Wittman & $\begin{array}{l}\text { The Use of Design-of- } \\
\text { Experiments Methodology to } \\
\text { Optimize Polymer Capsule } \\
\text { Fabrication }\end{array}$ \\
\hline Irene Lippa & Byron-Bergen & K. Marshall & $\begin{array}{l}\text { Synthesis and Analysis of } \\
\text { Nickel Dithiolene Dyes in a } \\
\text { Nematic Liquid Crystal Host }\end{array}$ \\
\hline $\begin{array}{l}\text { Phillip } \\
\text { Ostromogolsky }\end{array}$ & Brighton & F. Marshall & $\begin{array}{l}\text { Investigation of the X-Ray } \\
\text { Diffraction Properties of a } \\
\text { Synthetic Multilayer } \\
\end{array}$ \\
\hline $\begin{array}{l}\text { Miehael } \\
\text { Schubmehl }\end{array}$ & The Harley School & R. Epstein & $\begin{array}{l}\text { An Analysis of the Uncertainty } \\
\text { in Temperature and Density } \\
\text { Estimates from Fitting Model } \\
\text { Spectra to Data }\end{array}$ \\
\hline Joshua Silbermann & Penfield & P. Jannimagi & $\begin{array}{l}\text { Automated CCD Camera } \\
\text { Characterization }\end{array}$ \\
\hline Abigail Stern & The Harley School & J. Knauer & $\begin{array}{l}\text { Design and Testing of a } \\
\text { Compact X-Ray Diode }\end{array}$ \\
\hline Amy Turner & Churchville-Chili & R. S. Craxton & $\begin{array}{l}\text { Ray Tracing Through the Liquid } \\
\text { Crystal Point Diffraction } \\
\text { Interferometer }\end{array}$ \\
\hline
\end{tabular}


A total of 91 high school students have participated in the program since it began in 1989. The students this year were selected from approximately 60 applicants. Each applicant submitted an essay describing their interests in science, a copy of their transcript, and a letter of recommendation from a science or math teacher.

LLE plans to continue this program in future years. The program is strictly for students from Rochester-area high schools who have just completed their junior year. Applications are generally mailed out in February with an application deadline near the end of March. For more information about the program or an application form, please contact Dr. R. Stephen Craxton at LLE.

This program was supported by the U.S. Department of Energy Office of Inertial Confinement Fusion under Cooperative Agreement No. DE-FC03-92SF19460. 
AN ANALYSIS OF THE UNCERTAINTY IN TEMPERATURE AND DENSITY ESTIMATES FROM FITTING MODEL SPECTRA TO DATA

by

MICHAEL SCHUBMEHL

The Harley School

Rochester, NY 
An Analysis of the Uncertainty in Temperature and

Density Estimates from Fitting Model Spectra to Data

Michael P. Schubmehl

Advised by Dr. Reuben Epstein

Laboratory for Laser Energetics

University of Rochester

Summer High School Academic Research Program

August 26, 1998

\begin{abstract}
:
Temperature and density histories of direct-drive laser fusion implosions are important to an understanding of the reaction's progress. Such measurements also document phenomena such as preheating of the core and improper compression that can interfere with the thermonuclear reaction. Model $x$-ray spectra from the non-LTE (local thermodynamic equilibrium) radiation transport post-processor for LILAC have recently been fitted to OMEGA data. The spectrum fitting code reads in a grid of model spectra and uses an iterative weighted least-squares algorithm to perform a fit to experimental data, based on user-input parameter estimates. The purpose of this research was to upgrade the fitting code to compute formal uncertainties on fitted quantities, and to provide temperature and density estimates with error bars. A standard error-analysis process was modified to compute these formal uncertainties from information about the random measurement error in the data. Preliminary tests of the code indicate that the variances it returns are both reasonable and useful.
\end{abstract}

\title{
Objective:
}

The only item not addressed in the fitting code as it stands is error analysis. Formal uncertainties are not computed on fitted quantities, and thus how "good" the fit is can only be measured in a qualitative way. The goal of this research was to outline a method to compute the uncertainties on fitted quantities in a more formal and accurate way. In designing such a method, one must pay close attention to the exact nature of the errors that go into the fitting code. First, it was assumed that all systematic errors had been removed from the data prior to the beginning of the fitting process. Thus, the remaining error was entirely due to noise, either from the detector or the recording system. The exact properties of this random noise had to be determined based on knowledge of the instrument and the signal. For example, it is important to take into account that some errors may be correlated; that is, the errors at two nearby points may not be independent. Finally, this information had to be combined into an outline of a formal method for determining the error on all fitted quantities, including temperature and density. 


\section{Introduction:}

Direct-drive inertial confinement fusion relies on the uniform illumination of a spherical target, only about a millimeter in diameter, by an array of high-power laser beams. The target is composed of an outer shell of $\mathrm{CH}$ plastic that encapsulates a gaseous mix of deuterium and tritium. For the purposes of $x$-ray spectroscopy, the core is often doped with one-quarter to five percent (by pressure) argon gas, which produces diagnostic $x$-rays under fusion conditions. When the laser pulse strikes the plastic shell, it rapidly ablates, producing a tremendous inward force on the DT core. The rapid compression of the core results in an increase in both temperature and density, to the point where the electrostatic repulsion between deuterons and tritons is overcome by their kinetic energy, and the strong nuclear force takes over. The two nuclei fuse, releasing an alpha particle and a thermal neutron. Most of the energy produced in the reaction is carried off by these neutrons, which can then be collected. Ideally, most of the energetic alpha particles do not escape from the core, but collide with other deuterons and tritons, depositing their energy and propagating the reaction throughout the fuel mass.

The laser beams used in the experiment must be tuned extremely accurately, however. Nonuniformity in the illumination of the target can cause it to compress improperly, and snuff out the reaction. Also, the beams must be adjusted so as to minimize any preheating of the core due to the production of suprathermal electrons. Any such preheating greatly reduces the efficiency of the reaction, because it prevents the core from being properly compressed.

An accurate method for obtaining temperature and density histories makes it possible to monitor these effects. One such method is time-resolved $x$-ray spectroscopy of an argondoped core. The core, when doped with as little as one quarter of one percent argon (by

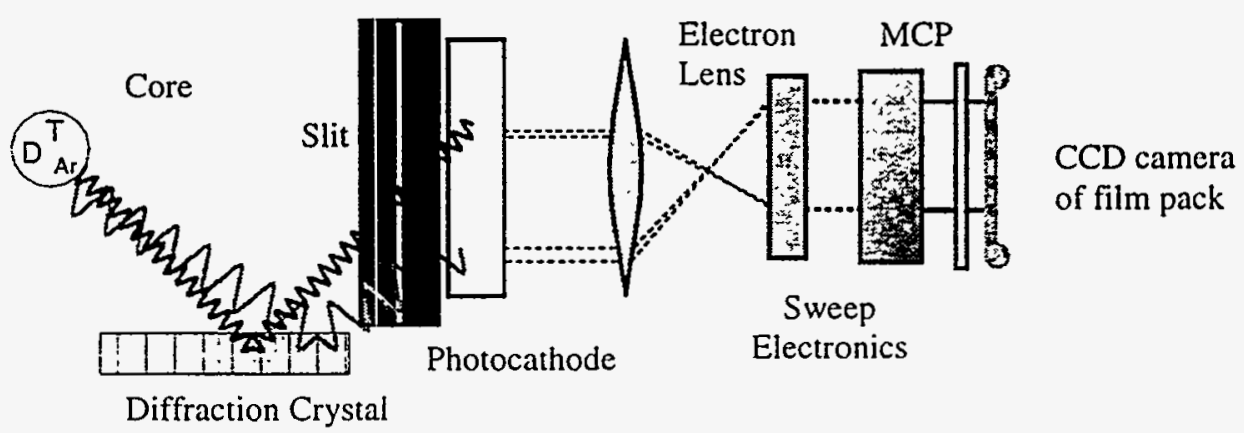

Figure $1 \mathrm{X}$-ray streak camera pressure) produces $\mathrm{x}$-rays of energies varying from just a few $\mathrm{keV}$ to around $100 \mathrm{keV}$. The intensity of this radiation can be measured with an x-ray streak camera, called an SSC (figure 1,) which is sensitive in a range from about 3.6-4.5 keV. X-rays from the source are Bragg-reflected off of a diffraction crystal, causing them to disperse into an energy spectrum, with higher energies on the top of the figure. This spectrum is passed through a narrow vertical slit, which turns it into a thin vertical bar. This thin vertical bar strikes a photocathode, which gives off electrons. These electrons are focused and passed to a time-dependent electric field that deflects the narrow vertical beam of electrons from left to right as time goes on. The signal is then passed to an MCP (microchannel plate) intensifier and strengthened. The sweep of this strengthened beam is recorded on film or a CCD camera, giving intensity information over a range of energies and a range of times. If the spectrum is recorded on film, it is then digitized and stored (figure 2.) The spectrum produced by this process is said to be "time-resolved" because it is essentially an x-ray history of the 
implosion. The easiest way to examine this history is to look at lineouts - that is, plots of intensity vs. photon energy at a particular time.

In order to understand the analysis of these lineouts, it is important to understand a little bit about the processes behind their formation. X-ray photons are produced by electron transitions within ions or the capture of an electron by an ion. The energy (and thus wavelength and frequency) of the photon produced by a transition is proportional to the energy separation between its initial and final energy states. Bound-bound emission, free-bound emission, and bound-bound absorption all effect the shapes of spectra. Let us first discuss bound-bound emission, which is the most important type of emission for the purposes of spectral analysis. At the temperatures necessary for nuclear fusion, argon is highly ionized, usually retaining only' a few electrons. Thus, it can undergo hydrogen- and helium-like electron transitions, producing $\mathrm{x}$-ray photons of energies given approximately by the formula below, with $Z_{\text {nuc }}$ equal to the screened nuclear charge, and $n_{\text {initial }}$ and $n_{\text {final }}$ equal to the primary quantum numbers of the initial and final states.

$$
E_{n}=\frac{13.6 \times Z_{n u c}^{2}}{n_{\text {final }}^{2}}-\frac{13.6 \times Z_{\text {nuc }}^{2}}{n_{\text {inirial }}^{2}}
$$

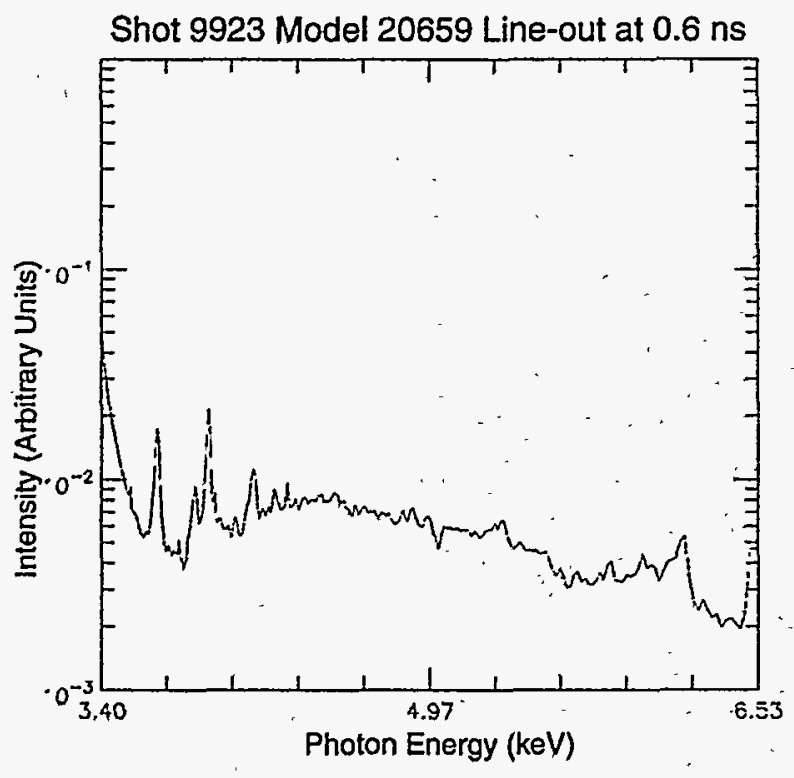

Figure 3 Lineout of shot 9923 TRS at $0.6 \mathrm{~ns}$
Time-Resolved Spectrum for Shot 9923

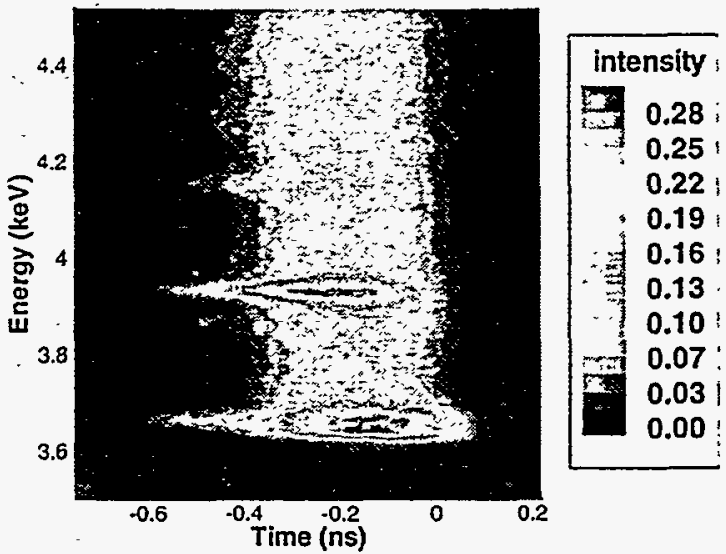

Figure 2 Time-resolved spectrum 


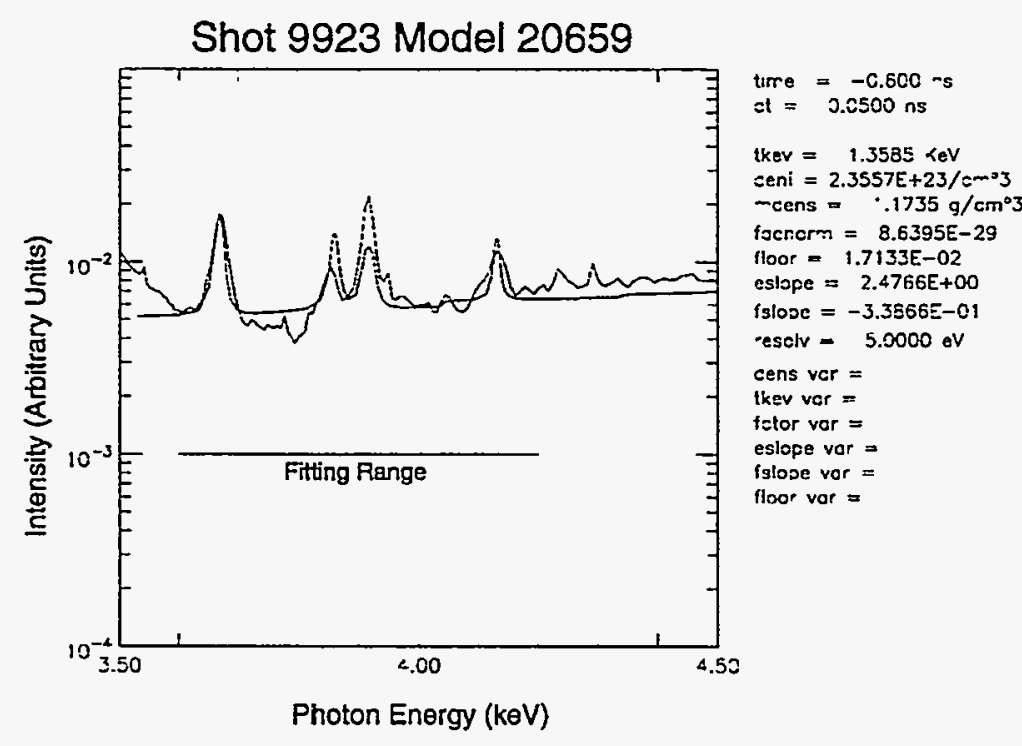

Figure 4 Sample spectrum - data and model. states are unstable, however, and a new photon may be re-emitted a short time later. This new photon may have a slightly different energy, because of perturbations of the upper and lower energy states by electrons in the near environment, however. As a result, the observed peaks can have modified line shapes, depending on the exact conditions in the core and its surrounding layers.

What makes it possible to determine temperatures and densities from the spectra formed by the above processes is the fact

that the number of $x$-rays produced at different energies is strongly dependent on the temperature, or average electron kinetic energy, of the core plasma and the density of electrons. In particular, the presence of high-velocity electrons in motion near quasi-static argon ions may perturb the argon's energy levels over time. Such fluctuations result in the production of x-rays of slightly different energies and contribute to the width of spectral lines. These perturbations are primarily density-dependent; greater ion densities (and, equivalently, greater mass densities) result in more perturbations and greater line-widths. Thus, by examining line width, it is possible to determine density. The ratios of lines at different temperatures also exhibit density dependence, but have an even stronger temperature dependence. Thus it is possible to determine the approximate temperature and density of the core at a particular point in time by examining the widths and relative heights of the lines in an argon x-ray spectrum. Since enough is known about the effects of temperature and density on line shapes to model them. comparison between model spectra and experimental data can produce estimates of temperature and density.

The model spectra currently used for fitting at LLE are generated by the non-LTE radiation transport post-processor for LILAC. in the form of a grid of model spectra. That is, given data on core composition and laser parameters, the post-processor's test mode calculates the $\mathrm{X}$-ray spectrum for a variety of temperatures and densities and stores them in a massive grid. This grid is then fed into the fitting program along with OMEGA data and initial estimates of temperature and density. After attempting some simple error correction, the fitting process begins with the selection of a time integration interval. Data points within this time interval are averaged by integration, in an attempt to eliminate some random error. Intervals are usually chosen to be sufficiently small (about 50 ps) that this averaging has no significant effect on the spectral features, other than reducing noise. The entire process can be repeated at different time intervals to get temperature and density histories. The next step in the program is the selection of an energy interval for viewing the new averaged data is set. This is important because all of the argon transitions fit for are in the range of 3.5 to $4.5 \mathrm{keV}$, the range over which the spectrometer is sensitive. The user then enters the intervals over which the model spectra are to be fitted to the 
data. These fit bands should be chosen such that all major spectral features (usually the He- $\beta$, He- $\gamma, \mathrm{H}-\beta$ and $\mathrm{H}-\gamma$ lines) are included in the fit.

The program also requires initial guesses for temperature and density in order to begin the iterative fit process. These are the first two parameters fitted for; as the code iterates, temperature and density estimates are made progressively better. If the initial guesses are reasonably accurate, the process converges quite quickly. The process is complicated, however, by the need to fit for other parameters as well.

The intensity values in the model spectra are computed in terms of the amount of energy that photons carry across a certain area in a certain amount of time, traveling in a direction confined to a certain solid-angle element. ${ }^{1}$ The corresponding intensities in OMEGA data, however, are in arbitrary units, because the streak camera system has never been formally calibrated. In order to avoid changing the data and still put the model and data intensities in the same range, the model is multiplied by an arbitrary constant. This constant is one of the parameters fitted for in the code. Another parameter is the floor intensity of the model. Because the fit to line shapes and line ratios is of primary interest, an arbitrary constant is added to the model to bring its floor up (or down) to the level of the data's background intensity. The fitting program also takes into account that this floor value may change over the selected energy range due to the continuum temperature. The floor value is thus assigned an exponential slope (which is also fitted for in the program) to allow the model to "tilt" in the same way the data does.

The fitting program produces graphical output after a set of initial guesses, and allows the user to refine these guesses before beginning the fitting process. Once the guesses are set to the user's satisfaction, the iterative fit process begins. The user selects which parameters to fit for in the first iteration, and the program returns new suggested values for those parameters. Only a fraction of this correction is taken, to avoid overshooting the "best" fit due to the linear approximations made by the program. This process is iterated until the fit is to the user's satisfaction. After completion, the program prints out data on the fit, including a logarithmic plot of the data and the model, the values of all parameters, and now formal estimates of the error in each parameter.

Procedures and Results:

Perhaps the simplest method of fitting a model to data is the least-squares method. In this method of fitting, the goal is to minimize a quantity called the chi-squared, defined as follows:

$$
\chi^{2}=\sum_{i=1}^{N}\left(f\left(x_{1}, x_{2}, \cdots, x_{p} ; t_{i}\right)-y_{i}\right)^{2}=\sum_{i=1}^{N}\left(\varepsilon_{i}\right)^{2}
$$

The method for doing this is to compute the partial derivatives of the chi-squared function with respect to each of the parameters $x_{1}, x_{2}$, etc. and use Newton's method to predict the values of the parameters that give zero partials. The changes that need to be made to the parameter values are

\footnotetext{
' Pọmraning, C,G, The Equations of Radiation Hydrodynamics. Pergamon Press, Oxford.
} 
computed simultaneously, using matrices. Because simple least-squares fitting treats each data point with equal weight, though, there is no way to express the fact that some data points are more reliable than others. To solve this problem. we introduce a weighting factor into the chisquared function:

$$
\chi^{2}=\sum_{i=1}^{N}\left(W_{i} \varepsilon_{i}^{2}\right)
$$

Although this is a better chi-squared to use in the fit, the problem of how to choose appropriate weights arises. In fact, the weights can be chosen automatically by using a method called maximum-likelihood weighted least-squares fitting. This method, currently used in the spectrumfitting code, determines the weight of a particular point from an estimate of the measurement error at that point. In order to discuss the process in more concrete terms, it is necessary to introduce matrix notation into the problem:

$\mathrm{y}$ is the $\mathrm{n} \times 1$ matrix of data points

$\mathrm{f}^{\circ}$ is an $\mathrm{n} \times 1$ matrix of nominal theoretical points, assumed to be close to $\mathbf{f}$

$\mathbf{f}$ is an $\mathrm{n} \times 1$ matrix of corrected theoretical points, depends on $\mathbf{x}$ $\varepsilon=(\mathbf{y}-\mathbf{f})$ is the $\mathrm{n} \times 1$ matrix of residuals

$\mathbf{S}=\left\langle\varepsilon_{\mathrm{i}} \times \varepsilon_{\mathrm{j}}\right\rangle^{\dagger}$ is the $\mathrm{n} \times \mathrm{n}$ covariance matrix of measurement errors

$\mathbf{x}$ is the $1 \times p$ matrix of parameters

$\mathbf{a}=\frac{\partial f_{f}}{\partial x_{r}}$ is the $n \times p$ matrix of partial derivatives

We now want to minimize:

so

and eventually

$$
\begin{gathered}
\chi^{2}=\sum_{i j}\left\lfloor\left(y_{1}-f_{i}\right)\left(S^{-1}\right)_{i j}\left(y_{j}-f_{j}\right)\right], \\
\frac{\partial}{\partial x}\left(\sum_{i j}\left[\left(y_{i}-f_{i}\right)\left(S^{-1}\right)_{i j}\left(y_{j}-f_{j}\right)\right]\right)=0 \\
\sum_{i j}\left[\left(a_{i k}\left(f_{j}-y_{j}\right)+a_{j k}\left(f_{i}-y_{i}\right)\right) \cdot\left(S^{-1}\right)_{i j}\right]=0
\end{gathered}
$$

Now we can use the fact that $f_{i}=f_{i}{ }^{\circ}+\sum_{m=1}^{p}\left(a_{i m} \times \delta x_{m}\right)$, where $\delta \mathrm{x}_{\mathrm{m}}$ is the correction to the $\mathrm{m}^{\text {th }}$ parameter from the nominal value to the corrected value; this quantity will ultimately be solved for. Using the approximation to $f_{i}$ given above, and assuming that $f_{i}$ and $f_{i}{ }^{\circ}$ are close enough that their partial derivatives with respect to each parameter are approximately equal, we have:

$$
\sum_{i j}\left[\left(a_{i k}\left(f_{j}^{\circ}+\sum_{m=1}^{p}\left(a_{j m} \times \delta x_{m}\right)-y_{j}\right)+a_{j k}\left(f_{i}^{\circ}+\sum_{m=1}^{p}\left(a_{i m} \times \delta x_{m}\right)-y_{i}\right)\right) \cdot\left(S^{-1}\right)_{i j}\right]=0
$$

\footnotetext{
${ }^{\prime}\langle\rangle$ denotes the expected value of a quantity over many trials.
} 
Because $S$ is symmetrical, $\mathbf{S}^{-1}$ is symmetrical, and thus one of the terms above can be eliminated and the entire expression can be manipulated to yield a simple equation of the form [Coefficients] $\times \delta \mathrm{x}=$ [Residuals], as follows:

$$
\begin{aligned}
\underline{C} \times \underline{\delta x} & =\underline{R} \\
& \underline{\left(a^{T} S^{-1} a\right)} \times \underline{\delta x}=\underline{\left(-a^{T} S^{-1}\left(f^{\circ}-\underline{y}\right)\right)}
\end{aligned}
$$

Thus, we can compute the values of the needed changes in the parameters to move from the initial estimate to a better one. The process can then be reapplied with this new estimate. These equations represent a slightly different way of solving for the new parameters than the one currently used in the code, however. In fact, this new method offers two significant advantages over the old method. First, it is compact, and allows all of the parameters to be solved for in a single step (with very few lines of code), given values for the other matrices. Most of the input matrices are simple to find, but the computation of the inverse covariance matrix $S^{-1}$. is somewhat nontrivial, and will be discussed in a moment. In fact, the very idea that errors at nearby points might not be independent is not addressed in the existing code. This change is the second major advantage to the new method. The only major drawback of the new method, compared to the older loop-oriented code, is that it requires significantly more space in main memory. Considering the wide availability of machines with large amounts of memory, however, this problem is of diminishing importance.

The computation of the inverse covariance matrix of the measurement errors $\mathbf{S}^{-1}$ was the next step. If we define a quantity called the correlation length $\lambda$, it seems obvious that the correlation between the errors at two points should decrease as the number of correlation lengths separating them increases. For the purposes of computation, it was assumed that the correlation between points separated by a distance $D$ was of the form:

$$
\begin{gathered}
\langle\varepsilon(x) \mathcal{\varepsilon}(x+D)\rangle=\varepsilon_{0}{ }^{2} e^{-D / \lambda} \\
\text { where } \varepsilon_{0}{ }^{2}=\sqrt{\left\langle\varepsilon(\dot{x})^{2}\right\rangle\left\langle\varepsilon(x+D)^{2}\right\rangle}
\end{gathered}
$$

However, the data put into the fitting program has been time- and energy-averaged by the very nature of the recording mechanism in most cases. Since spectra are usually recorded on film, the intensity value returned by the digitization process is actually an average over a certain time interval and a certain energy interval. It is possible to show ${ }^{2}$ that the correlation function between two such averaged blobs, where $\Delta$ is the time or energy interval, is of the form:

\footnotetext{
${ }^{2}$ Dr. Reuben Epstein. Private communication.
} 


$$
\begin{aligned}
\langle\overline{\varepsilon(x)} \cdot \overline{\varepsilon(x+D)}\rangle & =\frac{4 \lambda^{2} \varepsilon_{0}^{2}}{\Delta^{2}} e^{-D / \lambda} \sinh \left(\frac{\Delta}{2 \lambda}\right) \\
\left\langle\overline{\varepsilon(x)^{2}}\right\rangle & =2 \varepsilon_{0}^{2}\left(\frac{\lambda}{\Delta}\right)\left[1-\left(\frac{\lambda}{\Delta}\right)\left(1-e^{-\Delta / \lambda}\right)\right] \\
\left\langle\varepsilon(x)^{2}\right\rangle & =\frac{2 \varepsilon_{0}^{2}}{n_{c o r r}}, \text { where } n_{\text {corr }}=\Delta / \lambda
\end{aligned}
$$

The shot used for the purposes of this project, OMEGA shot 9923, is unusual, however: it was recorded directly onto a CCD camera, rather than onto film. Thus, it seems reasonable to use equations (2) rather than equations (3) to compute covariances for shot 9923 data, because there is no film involved.

Before using the equations above to compute the elements of $\mathrm{S}^{-1}$ it is necessary to discuss two matters. First. is it necessary to take covariance into account, and second, how are the measurement errors at a given point determined? The answers to these questions arise from an understanding of the instrument that takes the time-resolved spectra, and the errors it introduces. There are two main sources of error in the spectra: the detector, and the recording device. In the detector, there are several devices that may introduce error. First, the source of the $\mathrm{x}$-rays should ideally be a point source from the viewpoint of spectroscopy. It does have some size, however (about $100 \mu \mathrm{m}$,) and thus what should be a single sharp line is somewhat diffuse. Next, the $\mathrm{x}$-rays are reflected off an imperfect diffraction crystal that could cause identical $x$-rays to be reflected at slightly different angles. This signal is eventually focused by an electron lens. The lens itself is not perfect. however. and what should be a sharp signal can be defocused out to $100 \mu \mathrm{m}$. Finally, the signal is fed into the MCP intensifier, which damps the features of the signal to some degree. All in all, the detector produces a signal-to-noise ratio on the order of 100:1 in the data (at typical signal levels), but can also produce large correlated errors. In other words, the detector produces relatively little random noise, but some combination of more consistent errors can produce largescale correlated errors in the data. Most of the random noise comes from the recording process. ${ }^{3}$

Because the data is usually recorded on film, which has some degree of graininess, the data is corrupted by random. uncorrelated noise from film grain. The signal-to-noise ratio of the film is typically on the order of 10:1, and thus has a larger amplitude than the noise from the detector. This distinction gives rise to a way of determining whether covariance computations are necessary: simply look at the RMS (root-mean-square) amplitude of the noise over a smooth part of the spectrum and compare it to the maximum value that could be produced by uncorrelated film-grain error. If the noise is greater than this cutoff, it is probably correlated error from the detector, and a more detailed analysis must be performed. This test has not yet been implemented in the fitting code, but more exact signal-to-noise ratios are computable from the density wedges taken in most shots. In order to simplify the matter, the program assumes a signal-to-noise of 10 across all intensities: a more complete solution would be to analyze the density wedges for each shot to be fitted, and estimate the signal-to-noise ratio for that particular set of data. The appropriate method for analyzing the noise could then be selected based on whether the noise appeared to be correlated or uncorrelated.

\footnotetext{
${ }^{3}$ Dr. Paul Jaanimagi and Dr. David Bradley. Private communication.
} 
The final solution to the problem, then, is to compute the covariance matrix of the parameter estimates, whose diagonal is the variance of each parameter. It has been shown ${ }^{4}$ that this covariance matrix is simply the inverse of the coefficient matrix $\mathbf{C}$ in equations (1). By using equations (2) to compute the covariance matrix of the measurement error $S$, reasonable estimates of the variances of fitted parameters were obtained. The following curves are temperature and density plots over time, with the new formal error bars established by this research.

\section{Conclusions:}

The method described above is immediately useful, and yields interesting results that may warrant future analysis and exploration (figure 5.) It is interesting to note that the error bars appear to depend on the value of the temperature and density estimates; they are larger in the center of the density plot, and at the ends of the temperature plot. Due to the relatively little amount of data used, it would be premature to draw conclusions from this, however. The method will still benefit from some development, however. For example, the problem of how to automatically determine whether or not to take correlation into account is in itself nontrivial. Once such problems are solved, the spectrum-fitting code will provide even better variance estimates. The methods and equations presented above will be of immediate interest to the scientific community, however, because formal error bars on parameter estimates are not widely computed at present.
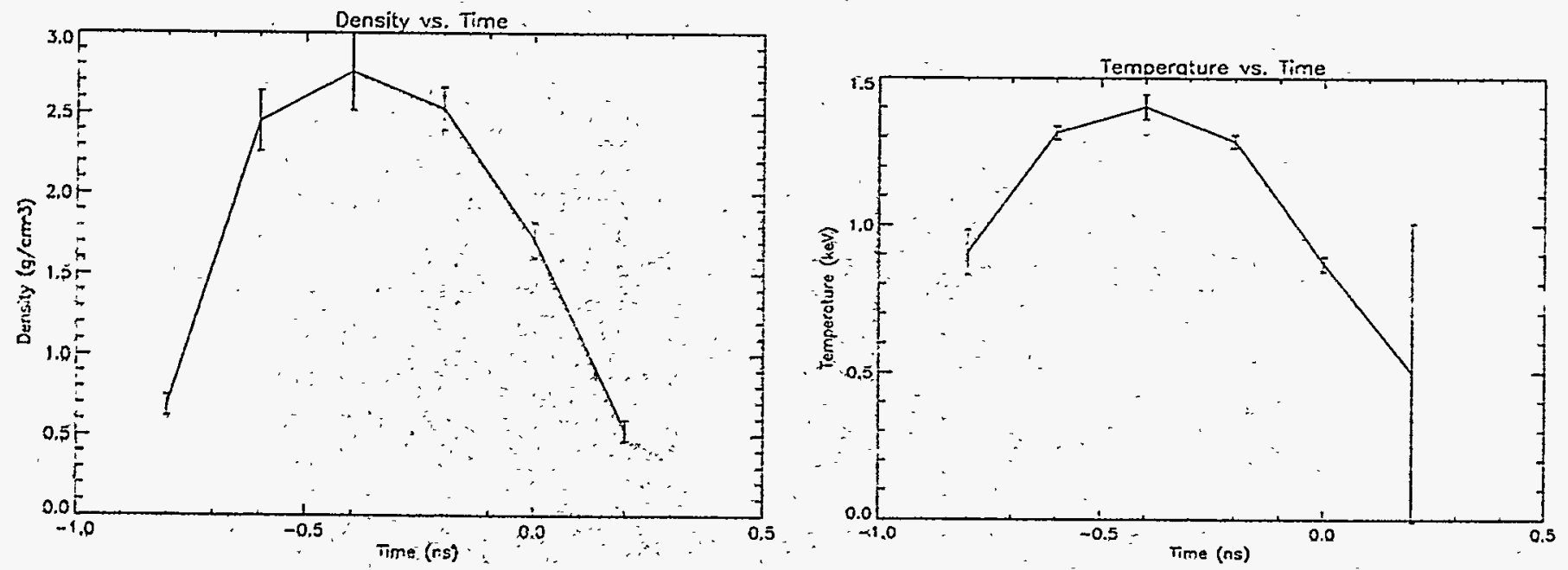

Figure 5 Denisity-time and temperature-time plots

\section{Acknowledgments;}

I would like to thank DrFDavid Bradiey, Dr. Paul Jaanimagi, and Dr. Donald Haynes for useful and instructive discussions of the material presented in this report, and Dr. Reuben Epstein for all his guidance and explanation throughout the project.

\footnotetext{
${ }^{4} \mathrm{Dr}$, Charles C Conselman III. Priyate communication.
} 